International Journal of Applied Mathematics

Volume 34 No. $3 \quad 2021,471-483$

ISSN: $1311-1728$ (printed version); ISSN: 1314-8060 (on-line version)

doi: http://dx.doi.org/10.12732/ijam.v34i3.4

\title{
UNIQUENESS OF DIFFERENCE DIFFERENTIAL POLYNOMIALS OF L-FUNCTIONS CONCERNING WEIGHTED SHARING
}

\author{
Nintu Mandal ${ }^{1}$, Nirmal Kumar Datta ${ }^{2}$ \\ ${ }^{1}$ Department of Mathematics \\ Chandernagore College, Chandernagore \\ Hooghly - 712136, West Bengal, INDIA \\ ${ }^{2}$ Department of Physics, Suri Vidyasagar College \\ Suri, Birbhum - 731101, West Bengal, INDIA
}

\begin{abstract}
In this paper, we mainly investigate the value distributions of difference differential polynomials of L-functions. Concerning small and rational functions sharing we prove uniqueness theorems on difference differential polynomials of L-functions. The results improve some recent results of W.J. Hao, J.F. Chen [3], W.Q. Zhu, J.F. Chen [17] and N. Mandal, N.K. Datta [10].
\end{abstract}

AMS Subject Classification: 11M36, 30D35

Key Words: meromorphic functions; L-functions; weighted sharing; uniqueness

\section{Introduction}

The Riemann hypothesis and its extension to the general classes of L-functions is the most important open problem in today's pure mathematics. In the modern number theory the L-functions play very important role.

$L(z)=\sum_{n=1}^{\infty} a(n) / n^{z}$ is said to be an L-function in the Selberg class if it satisfies the following assumptions:

(i) $a(n) \ll n^{\epsilon}$, for every $\epsilon>0$;

Received: September 21, 2020

(C) 2021 Academic Publications

${ }^{\S}$ Correspondence author 
(ii) There exists an integer $k \geq 0$ such that $(z-1)^{k} L(z)$ is a finite order entire function;

(iii) Every L-function satisfies the functional equation $\lambda_{L}(z)=\omega \overline{\lambda_{L}(1-\bar{z})}$, where $\lambda_{L}(z)=L(z) Q^{z} \prod_{i=1}^{k} \Gamma\left(\gamma_{i} z+\nu_{i}\right)$ with positive real numbers $Q, \gamma_{i}$ and complex numbers $\nu_{i}, \omega$ with $R e \nu_{i} \geq 0$ and $|\omega|=1$;

(iv) $L(z)=\prod_{p} L_{p}(z)$, where $L_{p}(z)=\exp \left(\sum_{k=1}^{\infty} b\left(p^{k}\right) / p^{k z}\right)$ with coefficients $b\left(p^{k}\right)$ satisfying $b\left(p^{k}\right) \ll p^{k \theta}$ for some $\theta<1 / 2$ and $p$ denotes prime number.

If $L$ satisfy only the assumptions (i)-(iii), then $L$ is an L-function in the extended Selberg class. In this paper by an $L$ function we always mean an $L$ function in the extended Selberg class with $a(1)=1$. Using Nevanlinna Value Distribution theory we study how uniquely difference differential polynomials of L-functions be determined in the extended Selberg class. We use the standard notations and definitions of the value distribution theory, [4].

Let $\alpha \in \mathbf{C} \cup\{\infty\}$ and $\xi, \psi$ be meromorphic functions in the complex plane. The set of all the $\alpha$ points of $\xi$ with multiplicities not exceeding $l$ is denoted by $E_{l)}(\alpha ; \xi)\left(\bar{E}_{l)}(\alpha ; \xi)\right)$, where $l$ is a positive integer and we consider(ignore) the multiplicities of the $\alpha$ points. We define the hyper order $\rho_{2}(\xi)$ of $\xi$ by $\rho_{2}(\xi)=\limsup _{r \rightarrow \infty} \frac{\log \log T(r, \xi)}{\log r}$. We denote by $S(r, \xi)$ any function satisfying $S(r, \xi)=o(T(r, \xi))$ as $r \longrightarrow \infty$, outside a possible exceptional set of finite linear measure. We say that $\xi$ and $\psi$ share $\alpha \mathrm{CM}$ if they have the same set of $\alpha$ points with the same multiplicities and if we do not consider the multiplicities then we say that $\xi$ and $\psi$ share $\alpha$ IM.

Definition 1. [7] Let $\xi$ be a meromorphic function defined in the complex plane. Let $n$ be a positive integer and $\alpha \in \mathbf{C} \cup\{\infty\}$. By $N(r, \alpha ; \xi \mid \leq n)$ we denote the counting function of the $\alpha$ points of $\xi$ with multiplicity $\leq n$ and by $\bar{N}(r, \alpha ; \xi \mid \leq n)$ the reduced counting function. Also by $N(r, \alpha ; \xi \mid \geq n)$ we denote the counting function of the $\alpha$ points of $\xi$ with multiplicity $\geq n$ and by $\bar{N}(r, \alpha ; \xi \mid \geq n)$ the reduced counting function. We define

$$
N_{n}(r, \alpha ; \xi)=\bar{N}(r, \alpha ; \xi)+\bar{N}(r, \alpha ; \xi \mid \geq 2)+\cdots+\bar{N}(r, \alpha ; \xi \mid \geq n) .
$$

Definition 2. [7] Let $\xi$ be a meromorphic function defined in the complex plane and $P(Z)$ be a small function of $\xi$ or rational function. Then we denote by $N(r, P ; \xi \mid \leq m), \bar{N}(r, P ; \xi \mid \leq m), N(r, P ; \xi \mid \geq m), \bar{N}(r, P ; \xi \mid \geq m), N_{m}(r, P ; \xi)$ etc. the counting functions $N(r, 0 ; \xi-P \mid \leq m), \bar{N}(r, 0 ; \xi-P \mid \leq m), N(r, 0 ; \xi-$ $P \mid \geq m), \bar{N}(r, 0 ; \xi-P \mid \geq m), N_{m}(r, 0 ; \xi-P)$ etc., respectively. 
Definition 3. $[5,6]$ Let $\xi$ and $\psi$ be two meromorphic functions defined in the complex plane and $n$ be an integer $(\geq 0)$ or infinity . we denote by $E_{n}(\alpha ; \xi)$ the set of all zeros of $\xi-\alpha$ where $\alpha \in \mathbf{C} \cup\{\infty\}$ and a zero of multiplicity $k$ is counted $k$ times if $k \leq n$ and $n+1$ times if $k>n$. we say that $\xi, \psi$ share $\alpha$ with weight $n$ if $E_{n}(\alpha ; \xi)=E_{n}(\alpha ; \psi)$.

We say $\xi, \psi$ share $(\alpha, n)$ to mean that $\xi, \psi$ share $\alpha$ with weight $n$. Clearly $\xi, \psi$ share $\alpha$ IM or CM if and only if $\xi, \psi$ share $(\alpha, 0)$ or $(\alpha, \infty)$, respectively.

In $2010 \mathrm{Li}$ [8] proved the following theorem.

Theorem 4. [8] Let $\xi$ be a nonconstant meromorphic function having finitely many poles and $L$ be a nonconstant $L$-function. If $\xi$ and $L$ share $(\alpha, \infty)$ and $(\beta, 0)$ then $L \equiv \xi$, where $\alpha$ and $\beta$ are two distinct finite values.

Definition 5. [10] Let $\xi$ be a meromorphic function defined in the complex plane and $P(z)$ be a rational function or a small function of $\xi$. Then we denote by $E_{m)}(P ; \xi), \bar{E}_{m)}(P ; \xi)$ and $E_{m}(P ; \xi)$ the sets $E_{m)}(0 ; \xi-P), \bar{E}_{m)}(0 ; \xi-P)$ and $E_{m}(0 ; \xi-P)$, respectively.

We write $\xi, \psi$ share $(P, n)$ to mean that $\xi-P, \psi-P$ share the value 0 with weight $n$. Clearly if $\xi, \psi$ share $(P, n)$ then $\xi, \psi$ share $(P, m)$ for all integers $m, 0 \leq m<n$. Also we note that $\xi, \psi$ share $P$ IM or CM if and only if $\xi, \psi$ share $(P, 0)$ or $(P, \infty)$, respectively.

Considering differential monomial in 2017, Liu, Li and Yi [9] proved the following uniqueness theorems.

Theorem 6. [9] Let $j \geq 1$ and $k \geq 1$ be integers such that $j>3 k+6$. Also let $L$ be an $L$-function and $\xi$ be a nonconstant meromorphic function. If $\left\{\xi^{j}\right\}^{(k)}$ and $\left\{L^{j}\right\}^{(k)}$ share $(1, \infty)$, then $\xi \equiv \alpha L$ for some nonconstant $\alpha$ satisfying $\alpha^{j}=1$.

Theorem 7. [9] Let $j \geq 1$ and $k \geq 1$ be integers such that $j>3 k+6$. Also let $L$ be an $L$-function and $\xi$ be a nonconstant meromorphic function. If $\left\{\xi^{j}\right\}^{(k)}(z)$ and $\left\{L^{j}\right\}^{(k)}(z)$ share $(z, \infty)$, then $\xi \equiv \alpha L$ for some nonconstant $\alpha$ satisfying $\alpha^{j}=1$.

Considering differential polynomials in 2018, W.J. Hao and J.F. Chen [3] obtained the following uniqueness results on L-function: 
Theorem 8. [3] Let $\xi$ be a nonconstant meromorphic function and $L$ be an $L$-function such that $\left[\xi^{n}(\xi-1)^{m}\right]^{(\tau)}$ and $\left[L^{n}(L-1)^{m}\right]^{(\tau)}$ share $(1, \infty)$, where $n, m, \tau \in \boldsymbol{Z}^{+}$. If $n>m+3 \tau+6$ and $\tau \geq 2$, then, $\xi \equiv L$ or, $\xi^{n}(\xi-1)^{m} \equiv$ $L^{n}(L-1)^{m}$.

Theorem 9. [3] Let $\xi$ be a nonconstant meromorphic function and $L$ be an L-function such that $\left[\xi^{n}(\xi-1)^{m}\right]^{(\tau)}$ and $\left[L^{n}(L-1)^{m}\right]^{(\tau)}$ share $(1,0)$, where $n, m, \tau \in \boldsymbol{Z}^{+}$. If $n>4 m+7 \tau+11$ and $\tau \geq 2$, then, $\xi \equiv \mathcal{L}$ or, $\xi^{n}(\xi-1)^{m} \equiv$ $L^{n}(L-1)^{m}$.

In 2019 W.Q. Zhu and J.F. Chen [17] using truncated sharing proved the following uniqueness theorem.

Theorem 10. [17] Let $L$ be an $L$-function and $\xi$ be a transcendental meromorphic function defined in the complex plane $C$. Also let $n, k(\geq 2)$, $l(\geq 2)$ be positive integers such that $n \geq 7 k+17$. If $\bar{E}_{l)}\left(1,\left(\xi^{n}(\xi-1)\right)^{(k)}\right)=$ $\bar{E}_{l)}\left(1,\left(L^{n}(L-1)\right)^{(k)}\right)$, then $f \equiv L$.

Considering truncated sharing of small functions in 2020 Mandal and Datta [10] proved the following theorem.

Theorem 11. [10] Let $L$ be a nonconstant $L$-function and $\rho$ be a small function of $L$ such that $\rho \not \equiv 0, \infty$. If $\bar{E}_{4)}(\rho ; L)=\bar{E}_{4)}\left(\rho ;\left(L^{m}\right)^{(k)}\right), E_{2)}(\rho ; L)=$ $E_{2)}\left(\rho ;\left(L^{m}\right)^{(k)}\right)$ and $2 N_{2+k}\left(r, 0 ; L^{m}\right) \leq(\sigma+o(1)) T(r, L)$, where $m \geq 1, k \geq 1$ are integers and $0<\sigma<1$, then $L \equiv\left(L^{m}\right)^{(k)}$.

Now the following questions come naturally.

Question 12. If we consider rational or small function sharing in Theorem 8, Theorem 9 and Theorem 10, then what happens?

Question 13. Can we take difference differential polynomials in place of differential polynomials in Theorem 8, Theorem 9, Theorem 10 and Theorem 11 ?

Definition 14. [5] Let two nonconstant meromorphic functions $\xi$ and $\psi$ share a value $\alpha$ IM. We denote by $\bar{N}_{*}(r, \alpha ; \xi, \psi)$ the counting function of the $\alpha$-points of $\xi$ and $\psi$ with different multiplicities, where each $\alpha$-point is counted 
only once.

Definition 15. Let two nonconstant meromorphic functions $\xi$ and $\psi$ share a value $\alpha$ IM. We denote by $\bar{N}(r, \alpha ; \xi \mid>\psi)$ the counting function of the $\alpha$-points of $\xi$ and $\psi$ with multiplicities with respect to $\xi$ is greater than the multiplicities with respect to $\psi$, where each $\alpha$-point is counted once only.

Definition 16. Let two nonconstant meromorphic functions $\xi$ and $\psi$ share a value $\alpha$ IM. We denote by $\bar{N}_{E}(r, \alpha ; \xi, \psi \mid>m)$ the counting function of the $\alpha$-points of $\xi$ and $\psi$ with multiplicities greater than $m$ and the multiplicities with respect to $\xi$ is equal to the multiplicities with respect to $\psi$, where each $\alpha$-point is counted once only.

In this paper we try to solve Questions 12, 13 and prove the following theorems.

Theorem 17. Let $L$ be a nonconstant $L$-function and $\xi$ be a transcendental meromorphic function. Let $\tau, n, \eta, \mu_{j}(j=1,2, \ldots, \eta), \lambda=\sum_{j=1}^{\eta} \mu_{j}$ be positive integers such that $n>\lambda+\eta(2 \tau+4)+4$ and $\omega_{j} \in \boldsymbol{C}-\{0\}(j=1,2, \ldots ., \eta)$ be distinct constants. Also let $\rho_{2}(L)<1, \rho_{2}(\xi)<1,\left[L^{n}(z) \prod_{j=1}^{\eta} L\left(z+\omega_{j}\right)^{\mu_{j}}\right]^{(\tau)}$ and $\left[\xi^{n}(z) \prod_{j=1}^{\eta} \xi\left(z+\omega_{j}\right)^{\mu_{j}}\right]^{(\tau)}$ share $(\rho(z), l)$ and $\xi, L$ share $(\infty, 0)$, where $\rho(z)$ is a small function of $\xi$ and $L$. If $l=0$ and $n>\lambda+(\eta+1)(5 \tau+7)$ or $l=1$ and $n>\lambda+\frac{3}{2}(\eta+1)(2 \tau+3)$, then one of the following holds:

(i) $\left[L(z)^{n} \prod_{j=1}^{\eta} L\left(z+\omega_{j}\right)^{\mu_{j}}\right]^{(\tau)} \equiv\left[\xi(z)^{n} \prod_{j=1}^{\eta} \xi\left(z+\omega_{j}\right)^{\mu_{j}}\right]^{(\tau)}$,

(ii) $\left[L(z)^{n} \prod_{j=1}^{\eta} L\left(z+\omega_{j}\right)^{\mu_{j}}\right]^{(\tau)}\left[\xi(z)^{n} \prod_{j=1}^{\eta} \xi\left(z+\omega_{j}\right)^{\mu_{j}}\right]^{(\tau)} \equiv \rho(z)^{2}$.

Theorem 18. Let $L$ be a nonconstant $L$-function and $\xi$ be a transcendental meromorphic function. Let $\tau, n, \eta, \mu_{j}(j=1,2, \ldots, \eta), \lambda=\sum_{j=1}^{\eta} \mu_{j}$ be positive integers such that $n>\lambda+\eta(2 \tau+4)+4$ and $\omega_{j} \in \boldsymbol{C}-\{0\}(j=1,2, \ldots, \eta)$ be distinct constants. Also let $\rho_{2}(L)<1, \rho_{2}(\xi)<1,\left[L^{n}(z) \prod_{j=1}^{\eta} L\left(z+\omega_{j}\right)^{\mu_{j}}\right]^{(\tau)}$ and $\left[\xi^{n}(z) \prod_{j=1}^{\eta} \xi\left(z+\omega_{j}\right)^{\mu_{j}}\right]^{(\tau)}$ share $(R(z), l)$ and $\xi$, L share $(\infty, 0)$, where $R(z)$ is a rational function. If $l=0$ and $n>\lambda+(\eta+1)(5 \tau+7)$ or $l=1$ and $n>\lambda+\frac{3}{2}(\eta+1)(2 \tau+3)$, then one of the following holds:

(i) $\left[L(z)^{n} \prod_{j=1}^{\eta} L\left(z+\omega_{j}\right)^{\mu_{j}}\right]^{(\tau)} \equiv\left[\xi(z)^{n} \prod_{j=1}^{\eta} \xi\left(z+\omega_{j}\right)^{\mu_{j}}\right]^{(\tau)}$,

(ii) $\left[L(z)^{n} \prod_{j=1}^{\eta} L\left(z+\omega_{j}\right)^{\mu_{j}}\right]^{(\tau)}\left[\xi(z)^{n} \prod_{j=1}^{\eta} \xi\left(z+\omega_{j}\right)^{\mu_{j}}\right]^{(\tau)} \equiv R(z)^{2}$. 


\section{Lemmas}

In this section we present some necessary lemmas.

Henceforth we denote by $\Omega$ the function defined by

$$
\Omega=\left(\frac{\Phi^{\prime \prime}}{\Phi^{\prime}}-\frac{2 \Phi^{\prime}}{\Phi-1}\right)-\left(\frac{\Psi^{\prime \prime}}{\Psi^{\prime}}-\frac{2 \Psi^{\prime}}{\Psi-1}\right) .
$$

Lemma 19. [12] Let $L$ be an $L$-function with degree $q$. Then $T(r, L)=$ $\frac{q}{\pi} r \log r+O(r)$.

Lemma 20. [10] Let $L$ be an $L$-function. Then $N(r, \infty ; L)=S(r, L)=$ $O(\log r)$.

Lemma 21. Let $\xi$ be a nonconstant meromorphic function and $L$ be an $L$-function. If $\xi$ and $L$ share $(\infty, 0)$, then $\bar{N}(r, \infty ; \xi)=S(r, L)=O(\log r)$.

Proof. Since $\xi$ and $L$ share $(\infty, 0)$, therefore by lemma 20 we have $\bar{N}(r, \infty ; \xi)=$ $\bar{N}(r, \infty ; L)=S(r, L)=O(\log r)$. This completes the proof.

Lemma 22. [16] Let $\xi(z)=\frac{\alpha_{0}+\alpha_{1} z+\ldots .+\alpha_{n} z^{n}}{\beta_{0}+\beta_{1} z+\ldots . .+\beta_{m} z^{m}}$ be a nonconstant rational function defined in the complex plane $\boldsymbol{C}$, where $\alpha_{0}, \alpha_{1}, \ldots, \alpha_{n}(\neq 0)$ and $\beta_{0}, \beta_{1}, \ldots, \beta_{m}(\neq 0)$ are complex constants. Then $T(r, \xi)=\max \{m, n\} \log r+$ $O(1)$.

Lemma 23. [13] Let $\xi$ be a transcendental meromorphic function of hyper order $\rho_{2}(\xi)<1$. Then for any $\alpha \in \boldsymbol{C}-0$ :

$$
\begin{gathered}
T(r, \xi(z+\alpha))=T(r, \xi(z))+S(r, \xi(z)), \\
N(r, \infty ; \xi(z+\alpha))=N(r, \infty ; \xi(z))+S(r, \xi(z)), \\
N(r, 0 ; \xi(z+\alpha))=N(r, 0 ; \xi(z))+S(r, \xi(z)) .
\end{gathered}
$$

Lemma 24. [11] Let $\Phi$ and $\Psi$ be two nonconstant meromorphic functions sharing $(1,1)$ and $(\infty, 0)$. If $\Omega \not \equiv 0$, then

$$
T(r, \Phi) \leq N_{2}(r, 0 ; \Phi)+N_{2}(r, 0 ; \Psi)+\frac{3}{2} \bar{N}(r, \infty ; \Phi)+\bar{N}(r, \infty ; \Psi)
$$




$$
\begin{aligned}
& +\bar{N}_{*}(r, \infty ; \Phi, \Psi)+\frac{1}{2} \bar{N}(r, 0 ; \Phi)+S(r, \Phi)+S(r, \Psi), \\
T(r, \Psi) \leq & N_{2}(r, 0 ; \Phi)+N_{2}(r, 0 ; \Psi)+\frac{3}{2} \bar{N}(r, \infty ; \Psi)+\bar{N}(r, \infty ; \Phi) \\
& +\bar{N}_{*}(r, \infty ; \Phi, \Psi)+\frac{1}{2} \bar{N}(r, 0 ; \Psi)+S(r, \Phi)+S(r, \Psi) .
\end{aligned}
$$

Lemma 25. [11] Let $\Phi$ and $\Psi$ be two nonconstant meromorphic functions sharing $(1,0)$ and $(\infty, 0)$. If $\Omega \neq \equiv$, then

$$
\begin{aligned}
T(r, \Phi) & \leq N_{2}(r, 0 ; \Phi)+N_{2}(r, 0 ; \Psi)+3 \bar{N}(r, \infty ; \Phi)+2 \bar{N}(r, \infty ; \Psi) \\
& +\bar{N}_{*}(r, \infty ; \Phi, \Psi)+2 \bar{N}(r, 0 ; \Phi)+\bar{N}(r, 0 ; \Psi) \\
& +S(r, \Phi)+S(r, \Psi), \\
T(r, \Psi) & \leq N_{2}(r, 0 ; \Phi)+N_{2}(r, 0 ; \Psi)+3 \bar{N}(r, \infty ; \Psi)+2 \bar{N}(r, \infty ; \Phi) \\
& +\bar{N}_{*}(r, \infty ; \Phi, \Psi)+2 \bar{N}(r, 0 ; \Psi)+\bar{N}(r, 0 ; \Phi) \\
& +S(r, \Phi)+S(r, \Psi) .
\end{aligned}
$$

Lemma 26. [15] Let $\Phi$ be a nonconstant meromorphic function and $k, p$ be two positive integers. Then

$$
\begin{gathered}
T\left(r, \Phi^{(k)}\right) \leq T(r, \Phi)+k \bar{N}(r, \infty ; \Phi)+S(r, \Phi), \\
N_{p}\left(r, 0 ; \Phi^{(k)}\right) \leq T\left(r, \Phi^{(k)}\right)-T(r, \Phi)+N_{p+k}(r, 0 ; \Phi)+S(r, \Phi), \\
N_{p}\left(r, 0 ; \Phi^{(k)}\right) \leq N_{p+k}(r, 0 ; \Phi)+k \bar{N}(r, \infty ; \Phi)+S(r, \Phi), \\
N\left(r, 0 ; \Phi^{(k)}\right) \leq N(r, 0 ; \Phi)+k \bar{N}(r, \infty ; \Phi)+S(r, \Phi) .
\end{gathered}
$$

Lemma 27. [2] Let $\xi$ be a transcendental meromorphic function of hyper order $\rho_{2}(\xi)<1$ and $\phi(z)=\prod_{j=1}^{\eta} \xi\left(z+\omega_{j}\right)^{\mu_{j}}$, where $n, \eta, \mu_{j}(j=1,2, \ldots, \eta), \lambda=$ $\sum_{j=1}^{\eta} s_{j}$ are positive integers and $c_{j} \in \boldsymbol{C}-\{0\}(j=1,2, \ldots, \eta)$ be distinct constants. Then

$$
(n-\lambda) T(r, \xi)+S(r, \xi) \leq T\left(r, \xi^{n} \phi\right) \leq(n+\lambda) T(r, \xi)+S(r, \xi) .
$$




\section{Proof of the Main Results}

\section{Proof of Theorem 17.}

Let $\phi(z)=\prod_{j=1}^{\eta} \xi\left(z+\omega_{j}\right)^{\mu_{j}}, \psi(z)=\prod_{j=1}^{\eta} L\left(z+\omega_{j}\right)^{\mu_{j}}, \Phi(z)=\frac{\left(\xi(z)^{n} \phi(z)\right)^{(\tau)}}{\rho(z)}$ and $\Psi(z)=\frac{\left(L(z)^{n} \psi(z)\right)^{(\tau)}}{\rho(z)}$. Then $\Phi, \Psi$ share $(1, l)$ and $\Phi, \Psi$ share $(\infty, 0)$ except for zeros and poles of $\rho(z)$.

Clearly by Lemma 19, $L$ is a transcendental meromorphic function. We have by Lemma 26 and Lemma 27:

$$
\begin{aligned}
N_{2}(r, 0 ; \Phi) & \leq N_{2}\left(r, 0 ;\left(\xi^{n} \phi\right)^{(\tau)}\right)+S(r, \xi) \\
& \leq T\left(r,\left(\xi^{n} \phi\right)^{(\tau)}\right)-T\left(r, \xi^{n} \phi\right)+N_{2+\tau}\left(r, 0 ; \xi^{n} \phi\right)+S(r, \xi) \\
& \leq T\left(r, \frac{\left(\xi^{n} \phi\right)^{(\tau)}}{\rho(z)}\right)-(n-\lambda) T(r, \xi)+N_{2+\tau}\left(r, 0 ; \xi^{n} \phi\right) \\
& +S(r, \xi) .
\end{aligned}
$$

Hence we get from (1)

$$
(n-\lambda) T(r, \xi) \leq T(r, \Phi)-N_{2}(r, 0 ; \Phi)+N_{2+\tau}\left(r, 0 ; \xi^{n} \phi\right)+S(r, \xi) .
$$

Similarly we get

$$
(n-\lambda) T(r, L) \leq T(r, \Psi)-N_{2}(r, 0 ; \Psi)+N_{2+\tau}\left(r, 0 ; L^{n} \psi\right)+S(r, L) .
$$

Now we have to consider the following two cases:

Case 1. Let $\Omega \not \equiv 0$. In this case we have to consider the following two subcases.

Subcase 1.1. Let $l=0$. Hence by Lemma 20, Lemma 21 and Lemma 25, we have from (2):

$$
\begin{aligned}
& (n-\lambda) T(r, \xi) \\
\leq & N_{2}(r, 0 ; \Phi)+N_{2}(r, 0 ; \Psi)+3 \bar{N}(r, \infty ; \Phi) \\
+ & 2 \bar{N}(r, \infty ; \Psi)+\bar{N}_{*}(r, \infty ; \Phi, \Psi)+2 \bar{N}(r, 0 ; \Phi) \\
+ & \bar{N}(r, 0 ; \Psi)-N_{2}(r, 0 ; \Phi)+N_{2+\tau}\left(r, 0 ; \xi^{n} \phi\right)+S(r, \xi)+S(r, L) \\
& \\
\leq & N_{2}(r, 0 ; \Phi)+N_{2}(r, 0 ; \Psi)+2 \bar{N}(r, 0 ; \Phi)+\bar{N}(r, 0 ; \Psi)
\end{aligned}
$$




$$
\begin{aligned}
& -N_{2}(r, 0 ; \Phi)+N_{2+\tau}\left(r, 0 ; \xi^{n} \phi\right)+S(r, \xi)+S(r, L) \\
& \leq N_{2}\left(r, 0 ;\left(\xi^{n} \phi\right)^{(\tau)}\right)+N_{2}\left(r, 0 ;\left(L^{n} \psi\right)^{(\tau)}\right)+2 \bar{N}\left(r, 0 ;\left(\xi^{n} \phi\right)^{(\tau)}\right) \\
& +\quad \bar{N}\left(r, 0 ;\left(L^{n} \psi\right)^{(\tau)}\right)-N_{2}\left(r, 0 ;\left(\xi^{n} \phi\right)^{(\tau)}\right)+N_{2+\tau}\left(r, 0 ; \xi^{n} \phi\right) \\
& +S(r, \xi)+S(r, L) \\
& \leq N_{2}\left(r, 0 ;\left(L^{n} \psi\right)^{(\tau)}\right)+2 \bar{N}\left(r, 0 ;\left(\xi^{n} \phi\right)^{(\tau)}\right)+\bar{N}\left(r, 0 ;\left(L^{n} \psi\right)^{(\tau)}\right) \\
& +N_{2+\tau}\left(r, 0 ; \xi^{n} \phi\right)+S(r, \xi)+S(r, L) \\
& \leq N_{2+\tau}\left(r, 0 ; L^{n} \psi\right)+2 N_{1+\tau}\left(r, 0 ; \xi^{n} \phi\right)+N_{1+\tau}\left(r, 0 ; L^{n} \psi\right) \\
& +N_{2+\tau}\left(r, 0 ; \xi^{n} \phi\right)+S(r, \xi)+S(r, L) \\
& \leq(2+\tau)(1+\eta) T(r, L)+2(\tau+1)(\eta+1) T(r, \xi) \\
& +(\tau+1)(\eta+1) T(r, L)+(2+\tau)(1+\eta) T(r, \xi) \\
& +S(r, \xi)+S(r, L) \\
& \leq(3+2 \tau)(1+\eta) T(r, L)+(3 \tau+4)(\eta+1) T(r, \xi) \\
& +S(r, \xi)+S(r, L) .
\end{aligned}
$$

Similarly by Lemma 20, Lemma 21 and Lemma 25, we have from (3):

$$
\begin{aligned}
(n-\lambda) T(r, L) & \leq(3+2 \tau)(1+\eta) T(r, \xi)+(3 \tau+4)(\eta+1) T(r, L) \\
& +S(r, \xi)+S(r, L) .
\end{aligned}
$$

Hence we get from (4) and (5)

$$
\begin{aligned}
(n-\lambda)\{T(r, L)+T(r, \xi)\} & \leq(7+5 \tau)(1+\eta)\{T(r, L)+T(r, \xi)\} \\
& +S(r, \xi)+S(r, L) .
\end{aligned}
$$

From $(6)$ we arrive at a contradiction, since $n>\lambda+(7+5 \tau)(1+\eta)$.

Subcase 1.2. Let $l=1$.

By Lemma 20, Lemma 21 and Lemma 24 we have from (2):

$$
\begin{aligned}
& (n-\lambda) T(r, \xi) \\
\leq & N_{2}(r, 0 ; \Psi)+\frac{3}{2} \bar{N}(r, \infty ; \Phi)+\bar{N}(r, \infty ; \Psi) \\
+ & \bar{N}_{*}(r, \infty ; \Phi, \Psi)+\frac{1}{2} \bar{N}(r, 0 ; \Phi)+N_{2+\tau}\left(r, 0 ; \xi^{n} \phi\right) \\
+ & S(r, \xi)+S(r, L) \\
\leq & N_{2}\left(r, 0 ;\left(L^{n} \psi\right)^{(\tau)}\right)+\frac{1}{2} N_{\tau+1}\left(r, 0 ; \xi^{n} \phi\right) \\
+ & \bar{N}\left(r, 0 ;\left(L^{n} \psi\right)^{(\tau)}\right)+N_{2+\tau}\left(r, 0 ; \xi^{n} \phi\right)+S(r, \xi)+S(r, L)
\end{aligned}
$$




$$
\begin{aligned}
& \leq(2+\tau)(1+\eta) T(r, L)+\frac{1}{2}(\tau+1)(\eta+1) T(r, \xi) \\
& +(2+\tau)(1+\eta) T(r, \xi)+S(r, \xi)+S(r, L) \\
& \leq(2+\tau)(1+\eta) T(r, L)+\frac{1}{2}(3 \tau+5)(\eta+1) T(r, \xi) \\
& +S(r, \xi)+S(r, L) .
\end{aligned}
$$

Similarly we have by Lemma 20, Lemma 21 and Lemma 24 from (3):

$$
\begin{aligned}
(n-\lambda) T(r, L) & \leq(2+\tau)(1+\eta) T(r, \xi)+\frac{1}{2}(3 \tau+5)(\eta+1) T(r, L) \\
& +S(r, \xi)+S(r, L) .
\end{aligned}
$$

Using (7) and (8) we get

$$
\begin{aligned}
(n-\lambda)\{T(r, L)+T(r, \xi)\} & \leq \frac{1}{2}(9+5 \tau)(1+\eta)\{T(r, L)+T(r, \xi)\} \\
& +S(r, \xi)+S(r, L) .
\end{aligned}
$$

Hence from (9) we arrive at a contradiction, since $n>\lambda+\frac{1}{2}(9+5 \tau)(1+\eta)$.

Case 2. Let $\Omega \equiv 0$. Then $\left(\frac{\Phi^{\prime \prime}}{\Phi^{\prime}}-\frac{2 \Phi^{\prime}}{\Phi-1}\right)-\left(\frac{\Psi^{\prime \prime}}{\Psi^{\prime}}-\frac{2 \Psi^{\prime}}{\Psi-1}\right) \equiv 0$.

Hence we have

$$
\Phi-1 \equiv \frac{\Psi-1}{b-c(\Psi-1)},
$$

where $b(\neq 0)$ and $c$ are constants.

Now we have to consider the following two cases.

Subcase 2.1. Let $c=0$. Then from (10) we have

$$
\Phi-1 \equiv \frac{(\Psi-1)}{b} .
$$

If $b \neq 1$, then from (11)

$$
\bar{N}(r, 0 ; \Phi)=\bar{N}(r, 1-b ; \Psi) .
$$

By Lemma 20, Lemma 26 and the second fundamental theorem, we have from (3)

$$
\begin{aligned}
& (n-\lambda) T(r, L) \\
= & T(r, \Psi)-N_{2}(r, 0 ; \Psi)+N_{\tau+2}\left(r, 0 ; L^{n} \psi\right)+S(r, L)
\end{aligned}
$$




$$
\begin{aligned}
& \leq \bar{N}(r, 0 ; \Psi)+\bar{N}(r, 1-b ; \Psi)+\bar{N}(r, \infty ; \Psi) \\
& -N_{2}(r, 0 ; \Psi)+N_{\tau+2}\left(r, 0 ; L^{n} \psi\right)+S(r, L) \\
& \leq \bar{N}(r, 0 ; \Psi)+\bar{N}(r, 0 ; \Phi)-N_{2}(r, 0 ; \Psi) \\
& +N_{\tau+2}\left(r, 0 ; L^{n} \psi\right)+S(r, L) \\
& \leq \bar{N}\left(r, 0 ;\left(\xi^{n} \phi\right)^{(\tau)}\right)+\bar{N}\left(r, 0 ;\left(L^{n} \psi\right)^{(\tau)}\right)+N_{\tau+2}\left(r, 0 ; L^{n} \psi\right)+S(r, L) \\
& \leq N_{\tau+1}\left(r, 0 ; \xi^{n} \phi\right)+N_{\tau+1}\left(r, 0 ; L^{n} \psi\right)+N_{\tau+2}\left(r, 0 ; L^{n} \psi\right)+S(r, L) \\
& \leq(\tau+1)(\eta+1) T(r, L)+(\tau+1)(\eta+1) T(r, \xi) \\
& +(\tau+2)(\eta+1) T(r, L)+S(r, L) \\
& \leq(2 \tau+3)(\eta+1) T(r, L)+(\tau+1)(\eta+1) T(r, \xi) \\
& +S(r, L)+S(r, \xi) .
\end{aligned}
$$

Similarly we have from (2)

$$
\begin{aligned}
(n-\lambda) T(r, \xi) & \leq(2 \tau+3)(\eta+1) T(r, \xi)+(\tau+1)(\eta+1) T(r, L) \\
& +S(r, L)+S(r, \xi) .
\end{aligned}
$$

From (13) and (14) we have

$$
\begin{aligned}
(n-\lambda)(T(r, L)+T(r, \xi)) & \leq(3 \tau+4)(\eta+1)(T(r, L) \\
& +T(r, \xi))+S(r, \xi)+S(r, L) .
\end{aligned}
$$

From (15) we arrive at a contradiction since $n>\lambda+(3 \tau+4)(\eta+1)$.

Hence $b=1$ and therefore we get from (11)

$$
\left[L^{n} \prod_{j=1}^{\eta} L\left(z+\omega_{j}\right)^{\mu_{j}}\right]^{(\tau)} \equiv\left[\xi^{n} \prod_{j=1}^{\eta} \xi\left(z+\omega_{j}\right)^{\mu_{j}}\right]^{(\tau)} .
$$

Subcase 2.2. Let $c \neq 0$ and $b=-c$.

If $c=1$, then from (10) we have $\Phi \Psi \equiv 1$. Hence

$$
\left[L^{n} \prod_{j=1}^{\eta} L\left(z+\omega_{j}\right)^{\mu_{j}}\right]^{(\tau)}\left[\xi^{n} \prod_{j=1}^{\eta} \xi\left(z+\omega_{j}\right)^{\mu_{j}}\right]^{(\tau)} \equiv \rho(z)^{2} .
$$

If $c \neq 1$, then from (10) we have $\frac{1}{\Phi}=\frac{-c \Psi}{(1-c) \Psi-1}$.

Hence $\bar{N}(r, 0 ; \Phi)=\bar{N}\left(r, \frac{1}{1-c} ; \Psi\right)$.

Now proceeding as in Subcase 2.1. we arrive at a contradiction. 
If $c=1$, then from (10) we have

$$
\Phi \equiv \frac{-b}{\Psi-b-1} .
$$

By Lemma 21 we have from (16)

$$
\bar{N}(r, C+1 ; \Psi)=\bar{N}(r, \infty ; \Phi)=\bar{N}(r, \infty ; \xi)+S(r, L)=S(r, L) .
$$

Now proceeding as in Subcase 2.1. we arrive at a contradiction.

If $c \neq 1$, then from (10) we have

$$
\Phi-\left(1-\frac{1}{c}\right) \equiv \frac{-b}{c^{2}\left(\Psi-\frac{b+c}{c}\right)} .
$$

Therefore by Lemma 21 we have

$$
\bar{N}\left(r, \frac{b+c}{c} ; \Psi\right)=\bar{N}(r, \infty ; \Phi)=\bar{N}(r, \infty ; \xi)+S(r, L)=S(r, L) .
$$

Hence proceeding as in Subcase 2.1. we arrive at a contradiction.

This completes the proof of the theorem.

\section{Proof of Theorem 18.}

Since $\xi$ and $L$ are transcendental meromorphic functions and $R(z)$ is a rational function therefore $R(z)$ is a small function of $\xi$ and $L$.

Hence by Theorem 17 we get the required result.

\section{References}

[1] A. Banerjee, Uniqueness of meromorphic functions sharing two sets with finite weight, Portugal. Math. (N.S.), 65, No 1 (2008), 81-93; DOI: 10.4171/PM/1800.

[2] R.S. Dyavanal, A.M. Hattikal, On the uniqueness of product of difference polynomials of meromorphic functions, Konuralp J. Math., 4, No 2 (2016), 42-55.

[3] W.J. Hao and J.F. Chen, Uniqueness of L-functions concerning certain differential polynomials, Dis. Dyn. Nat. Soc., (2018), Art. ID 4673165, 12 pp.; DOI: 10.1155/2018/4673165. 
[4] W.K. Hayman, Meromorphic Functions, The Clarendon Press, Oxford (1964).

[5] I. Lahiri, Weighted sharing and uniqueness of meromorphic functions, Nagoya Math. J., 161 (2001), 193-206; DOI: 10.1017/S0027763000027215.

[6] I. Lahiri, Weighted value sharing and uniqueness of meromorphic functions, Complex Var. Theory Appl., 46 (2001), 241-253; DOI: 10.1080/17476930108815411.

[7] I. Lahiri, N. Mandal, Small functions and uniqueness of meromorphic functions, J. Math. Anal. Appl., 340 (2008), 780-792; DOI: 10.1016/j.jmaa.2007.09.017.

[8] B.Q. Li, A result on value distribution of L-functions, Proc. Amer. Math. Soc., 138 (2010) 2071-2077; DOI: 10.1090/S0002-9939-09-10222-8.

[9] F. Liu, X.M. Li, H.X. Yi, Value distribution of L-functions concerning shared values and certain differential polynomials, Proc. Japan Acad. Ser. A, 93 (2017), 41-46; DOI: 10.3792/pjaa.93.41.

[10] N. Mandal, N.K. Datta, Uniqueness of L-function and its certain differential monomial concerning small functions, J. Math. Comput. Sci., 10, No 5 (2020), 2155-2163; DOI: 10.28919/jmcs/4836.

[11] P. Sahoo, Meromorphic functions that share fixed points with finite weights, Bull. Math. Anal. Appl., 2 (2010), 106-118.

[12] J. Steuding, Value-distribution of L-functions, Spinger, Berlin (2007).

[13] X.Y. Xu, On the value distribution and uniqueness of difference polynomials of meromorphic functions, Adv. Diff. Equ., 90, No 1 (2013), 13 pp.; DOI: $10.1186 / 1687-1847-2013-90$.

[14] L. Yang, Normality for families of meromorphic functions, Sci. Sinica Ser. A, 29, No 12 (1986), 1263-1274.

[15] C.C. Yang, H.X. Yi, Uniqueness Theory of Meromorphic Functions, Kluwer Academic Publ., Dordrecht (2003); Chinese original: Science Press, Beijing (1995).

[16] L. Yang, Value Distribution Theory, Spinger-Verlag, Berlin (1993). 
[17] W.Q. Zhu, J.F. Chen, Differential polynomials of L-functions with truncated shared values, Open Math., 17 (2019), 1113-1125; DOI: 10.1515/math-2019-0087. 